\title{
Phase Transitions in KDP-Type Crystals
}

\author{
VIJAY SINGH BIST, NARAYAN SINGH PANWAR and BIRENDRA SINGH SEMWAL
}

University Science Instrumentation Centre, School of Engineering and Technology, HNB Garhwal University Srinagar (Garhwal) Uttrakhand - 24 6174, India

vsbist_usic68@yahoo.com

Received 18 September 2015 / Accepted 5 October 2015

\begin{abstract}
Curie temperature is determined by investigating the variation of order-parameter $\left\langle S_{q}^{L}>\right.$ with temperature. The model Hamiltonian proposed by Blinc and Zeks has been modified by considering the lattice anharmonicities up to fourth order. The correlations appearing in the dynamical equation have been evaluated using double-time thermal retarded Green's function and Dyson's equation. The expressions for shift, width, renormalized soft mode frequency, Curie temperature, the expectation value of the proton collective mode components at site $\mathrm{q}\left(<S_{q}^{Z}>\right.$, and $<S_{q}^{X}>$ ) have been derived and discussed in KDP - type crystals. By fitting model values of physical quantities, the temperature dependence of $\left\langle S_{q}^{L}\right\rangle$, and $\left\langle S_{q}^{X}\right\rangle$ for different value of four-body coupling have been calculated. The theoretical results are found in good agreement with the experimental results.
\end{abstract}

Keywords: Phase transition, Curie temperature, Green's function, Dyson's equation, Soft mode frequency

\section{Introduction}

It was first shown by $\mathrm{Bush}^{1}$ that potassium dihydrogen phosphate, $\mathrm{KDP}\left(\mathrm{KH}_{2} \mathrm{PO}_{4}\right)$, exhibits a phase transition at low temperature. KDP is prototype of a family of crystals with bridging hydrogen bonds and its physical properties have been extensively studied ${ }^{2-13}$. Above the Curie temperature $\left(\mathrm{T}_{\mathrm{c}}\right)$, KDP has tetragonal structure belonging to space group $I \overline{4} 2 d\left(D_{2 d}^{12}\right)$. The orthorhombic phase belongs to space charge group Fdd2 $\left(C_{2 V}^{19}\right)$ and exits at room temperature lower than $\mathrm{T}_{\mathrm{c}}=123 \mathrm{~K}$. In the ferroelectric phase the positions of all hydrogen are fixed to $\mathrm{PO}_{4}$ radicals and a spontaneous polarization appears along the c-axis, and is not attributed directly to hydrogen, but to the displacement of $\mathrm{K}^{+}$ions and deformation of the tetrahedra of $\mathrm{PO}_{4}$ radicals which are induced by the ordered arrangement of hydrogen. In the paraelectric phase the hydrogen atoms moves between two equivalent equilibrium 
positions in the O-H- -O hydrogen bonds, linking the $\mathrm{PO}_{4}$ tetrahedral, and the configuration of hydrogen is random and the spontaneous polarization disappears. Below $\mathrm{T}_{c}$ this motion freezes out and the structure orders. This ordering is gradual and accompanied by displacement of the heavy atoms ${ }^{14}$. The real nature of the ferroelectric phase transition and the isotope effect on Tc for $H \rightarrow D$ exchange in KDP-type ferroelectrics has not been fully explained ${ }^{14-16}$. The proton tunneling mode ${ }^{17}$, the earliest model used to explain the isotope effect, predicted the displacive type of phase transition. More recent crystal lattice dynamics measurements, on the other hand, have suggested the order-disorder mechanism of phase transition, an extensively discussed elsewhere ${ }^{15-}$ ${ }^{16}$. Raman spectroscopic studies confirm that the ferroelectric phase transition in KDP $\left(\mathrm{KH}_{2} \mathrm{PO}_{4}\right)$, DKDP $\left(\mathrm{KD}_{2} \mathrm{PO}_{4}\right)$ and their mixed crystals is due to the "order-disorder dynamics" of $\mathrm{PO}_{4}$ dipoles ${ }^{8}$.

Kaminow and Damen ${ }^{18}$ first observed the soft mode associated with the ferroelectric phase transition of the KDP-type crystals at $122.3 \mathrm{~K}$ by measuring the low frequency Raman scattering in $\mathrm{x}(\mathrm{yx}) \mathrm{y}$ configuration. Since then $\mathrm{B}_{2}(Z)$ soft mode, which is connected to the susceptibility along the crystalline c-axis through the Lyddane-SachsTeller relation ${ }^{19}$, has been extensively studied by $\operatorname{Scott~}^{20}$ and interpreted using the pseudospin model $^{17,21,22}$ and its modifications ${ }^{6,23}$. In these theories, such a particular mode of proton motions along hydrogen bonds in a-b plane is coupled to other ion modes, bearing an electrical dipole moment along c-axis, is considered to play an essential role for the ferroelectric transition and therefore little attention has been paid to the modes other than $\mathrm{B}_{2}(\mathrm{Z})$ soft mode. In KDP crystals, however, there are four tunneling protons in a primitive unit cell of the paraelectric phase and consequently four normal modes are belonging to a $\mathrm{B}_{2}(\mathrm{Z})$, a doubly degenerate $\mathrm{E}(\mathrm{x}, \mathrm{y})$ and $\mathrm{A}_{2}$ modes $^{24}$. The $\mathrm{E}(\mathrm{x}, \mathrm{y})$ mode, which is both infrared and Raman active, provides valuable information, because this mode as well as $\mathrm{B}_{2}(\mathrm{Z})$ mode, reflect the nature of collective proton motion which triggers the phase transition, and moreover, in contrast to $\mathrm{B}_{2}(\mathrm{Z})$, it is directly related to the polarization along the crystalline a-axis.

At first, $\mathrm{Pak}^{25}$ employed Green's function methods in the order-disorder type ferroelectrics, who however, did not consider the anharmonic interactions. The phonon anharmonic interactions have been found very important in explaining dielectric, thermal and scattering properties of solids by many authors ${ }^{9-13,26}$ in the past. Pak's theory was further developed by Ramakrishanan and Tanaka ${ }^{27}$, who calculated the excitation spectrum of the system, but did not consider the anharmonic interactions. Their attempt, however, established the superiority of Green's function method over the other methods. Ganguli ${ }^{28}$ et al modified Ramakrishanan and Tanaka theory by considering anharmonic interaction. Their treatment explains many features of order-disorder ferroelectrics. However, due to insufficient treatment of anharmonic interactions, they could not explain quantitatively good results and could not describe some very interesting properties, like dielectric properties, acoustic attenuation, relaxation rate etc.

In the present study, the four- particle cluster model Hamiltonian with the phonon anharmonicity upto fourth order has been taken to theoretical study of phase transitions in KDP-type crystals, using the double time Green's function method and Dyson's equation. The proton Green's function and phonon Green's function have been solved for the collective system. Expressions for collective mode frequency shifts, widths, Curie temperature, and the expectation value of the proton collective mode components at site $\mathrm{q}\left(\left\langle S_{q}^{Z}\right\rangle\right.$, and $\left.\left\langle S_{q}^{X}\right\rangle\right)$ 
have been derived and discussed in KDP-type crystals. By fitting model values of physical quantities, the temperature dependence of $\left\langle S_{q}^{Z}\right\rangle$, and $\left\langle S_{q}^{X}\right\rangle$ for different value of fourbody coupling have been calculated. The theoretical results are found in good agreement with the experimental results.

\section{Theory of application to cluster model Hamiltonian}

For KDP crystals, the four - particle cluster model Hamiltonian ${ }^{14}$ alongwith third-and fourthorder phonon anharmonic interaction terms is expressed as:

$$
\begin{aligned}
H= & -2 \Omega \sum_{i} S_{i}^{X}-\frac{1}{2} \sum_{i j} J_{i j} S_{i}^{Z} S_{j}^{Z}-\frac{1}{4} \sum_{i j k l} J_{i j}^{\prime} S_{i}^{Z} S_{j}^{Z} S_{k}^{Z} S_{l}^{Z} \\
& +\frac{1}{4} \omega_{k}\left(A_{k}^{+} A_{k}+B_{k}^{+} B_{k}\right)-\sum_{i} \bar{V}_{i k} S_{i}^{X} A_{k} \\
& +\vec{k}_{1}, \sum_{2}, \vec{k}_{3} V_{3}\left(\vec{k}_{1} \vec{k}_{2} \vec{k}_{3}\right) A_{\vec{k}_{1}}, A_{\vec{k}_{2}}, A_{\vec{k}_{3}} \\
& +\vec{k}_{1}, \vec{k}_{2}, \vec{k}_{3}, \vec{k}_{4} V_{4}\left(\vec{k}_{1} \vec{k}_{2} \vec{k}_{3} \vec{k}_{4}\right) A_{\vec{k}_{1}}, A_{\vec{k}_{2}}, A_{\vec{k}_{3}}, A_{\vec{k}_{4}}
\end{aligned}
$$

where the first two terms constitute the original pseudo spin model Hamiltonian and the third is the quadrupole contribution (the four-body interaction). $S_{i}^{X}$ is the tunneling operator which measures the tunneling power of the proton between the hydrogen double well, $2 \Omega$ the tunneling frequency and $S_{i}^{Z}$ the half of the difference of the occupation probabilities for the proton in the two equilibrium positions of hydrogen bond. $J_{i j}$ is the two body coupling coefficient is the same for energy pair of protons in KDP and the four body coupling coefficient $J^{\prime} i j k l$ refers to the four hydrogen bonds in the $\mathrm{PO}_{4}$ group in $\mathrm{KDP}, \omega_{k}$ is bare phonon frequency: $A_{k}$ and $B_{k}$ are displacement and momentum operators. $\bar{V}_{i k}$ is protonlattice interaction term: $V_{3}$ and $V_{4}$ are the third- and fourth order anharmonic coefficients, defined by Maradudin et al. ${ }^{32}$.

\section{Proton Green's function:}

The proton Green's function $\quad<s_{q}^{Z}(t) ; s_{q^{\prime}}^{Z}\left(t^{\prime}\right)>>$ have been evaluated for the collective motion of the system, using model Hamiltonian Equation 1. The higher order correlations in the proton's Green's function have been evaluated using the symmetrical decoupling scheme, after applying the Dyson's treatment. With this approach, one gets

$$
\operatorname{Limit}_{\varepsilon \rightarrow 0} G_{q q^{\prime}}^{Z Z}(\omega+j \varepsilon)=\frac{\Omega<S_{q}^{X}>\delta_{q q^{\prime}}}{\pi\left[\omega^{2}-\widetilde{\Omega}^{2}+j \Gamma_{S}(q, \omega)\right]}
$$

Where $\widetilde{\widetilde{\Omega}}$ is the proton renormalized frequency of the coupled system, which on solving self consistently takes the form

$$
\widetilde{\Omega}^{2}=\widetilde{\Omega}^{2}+2 \Omega \Delta_{S}(q, \omega)
$$


$\Delta_{S}(q, \omega)$ and $\Gamma s(q, \omega)$ represent collective proton mode frequency shift and width respectively. The collective proton mode half width, is given by

$$
\begin{aligned}
\Gamma_{S}(q, \omega)= & \frac{-4 \pi \bar{V}_{q}^{2} \omega_{q}^{2}<S_{q}^{X}>\delta_{q q}^{\prime} \Gamma_{p}}{\Omega\left[\left(\omega^{2}-\widetilde{\widetilde{\omega}}_{q}^{2}\right)^{2}+4 \omega_{q}^{2} \Gamma_{p}^{2}\right]}+\frac{\pi b c^{2}}{2 \widetilde{\Omega}}\{\delta(\omega-\widetilde{\Omega})-\delta(\omega+\widetilde{\Omega}\} \\
& +\frac{\pi a^{2} \hat{\Omega}}{2 b}\{\delta(\omega-\hat{\Omega})-\delta(\omega+\hat{\Omega}\},
\end{aligned}
$$

The expectation value of the proton collective mode component at site ' $q$ ' have been obtained $^{33}$ as

$$
<S_{q}^{X}>=\frac{\Omega}{\widetilde{\Omega}} \tanh \left(\frac{\beta \widetilde{\Omega}}{2}\right), \quad<S_{q}^{y}>=0, \text { and }<S_{q}^{Z}>=\frac{a}{2 \widetilde{\Omega}} \tanh \left(\frac{\beta \widetilde{\Omega}}{2}\right)
$$

where

$$
\widetilde{\Omega}^{2}=a^{2}+b^{2}-b c
$$

with

$$
\begin{gathered}
\left.a=\sum_{q} J_{q}<S_{q}^{Z}>+\sum_{q} J_{q}^{\prime}<S_{q}^{Z}>^{3}\right) ; \\
b=2 \Omega ; \\
c=\sum_{q} J_{q}<S_{q}^{X}>+\underset{q}{+3 J_{q}^{\prime}}<S_{q}^{X}><S_{q}^{Z}>^{2}
\end{gathered}
$$

Equation 5 represents a system of $3 \mathrm{~N}$ equations for the average values of the collective mode components. The solution of this system will, however, be stable only if they minimize the free energy, i.e., if $\left\langle S_{q}^{Z}\right\rangle=\left\langle S_{q}^{y}\right\rangle=0$, and

$$
\text { so }<S_{q}^{X}>=\frac{\Omega}{\left(4 \Omega^{2}-2 \Omega \sum_{q}<S_{q}^{X}>\right)^{1 / 2}} \tanh \left(\frac{\left.4 \Omega^{2}-2 \Omega \sum_{q}<S_{q}^{X}>\right)^{1 / 2}}{q}\right) \text {, }
$$

Equation 5a, represent the paraelectric phase which exists at all temperature $(\mathrm{T}>\mathrm{Tc})$, and in the ferroelectric phase $(\mathrm{T}<\mathrm{Tc})^{31}$, as

$$
<S_{q}^{X}>=\frac{2 \Omega}{\sum_{q} J_{q}+\sum_{q^{\prime}} J_{{ }_{q}^{\prime}}<S_{q}^{Z}>^{2}}, \text { and }<S_{q}^{Z}>=\frac{1}{2} \tanh \left(\frac{a}{2 k_{B} T}\right)
$$

where $\beta=\left(k_{B} T\right)^{-1}, k_{B}$ is Boltzmann's constant and T the absolute temperature. 


\section{Phonon Green's function}

The acoustic mode frequency is obtained on solving phonon Green's function $<<A_{q} ; A_{q^{\prime}}^{+}>>$the higher order correlations in the phonon response have been calculated without decoupling and using renormalized Hamiltonian, as

$$
\widetilde{\widetilde{\omega}}_{q}^{2}=\widetilde{\omega}_{q}^{2}+2 \omega_{q} \Delta_{P}(q, \omega)
$$

with $\widetilde{\omega}_{q}^{2}=\omega_{q}^{2}+8 \omega_{q}\left(2 V_{3}+V_{4}\right) \operatorname{coth}\left(\frac{\beta \omega_{q}}{2}\right)$ calculating equation (6) self consistently and approximating, the collective mode frequency is given by

$$
\widetilde{\widetilde{\omega}}_{q \pm}^{2}=\frac{1}{2}\left(\widetilde{\omega}_{q}^{2}+\widetilde{\Omega}^{2}\right) \pm \frac{1}{2}\left[\left(\widetilde{\omega}_{q}^{2}+\widetilde{\widetilde{\Omega}}^{2}\right)^{2}+16 \bar{V}_{q}^{2} \omega_{q} \Omega<S_{q}^{X}>\right]^{1 / 2}
$$

\section{Transition temperature}

In the vicinity of transition (Curie) temperature in the paraelectric phase $\left\langle S_{q}^{L}>=0\right.$, one may expand $\widetilde{\widetilde{\omega}}_{q-}$ in the power of $\left(\mathrm{T}-\mathrm{T}_{\mathrm{c}}\right)$ around its value at $\mathrm{T}_{\mathrm{c}}$ getting immediately

$$
\begin{aligned}
\widetilde{\widetilde{\omega}}_{q-}^{2}= & \left(\frac{\partial \widetilde{\widetilde{\omega}}_{q-}^{2}}{\partial T}\right)_{T=T_{C}}\left(T-T_{C}\right) \\
& \widetilde{\widetilde{\omega}}_{q-}^{2} \cong \gamma \times\left(T-T_{C}\right) \text {; using Eq. (3) }
\end{aligned}
$$

where

$$
\gamma=\frac{\Omega^{2} \hat{J}}{\left[k_{B} T_{C}^{2} \cosh \left(\frac{\Omega}{k_{B} T_{C}}\right)\right]},
$$

with effective exchange coupling constant

and transition temperature

$$
\hat{J}=J_{0}+\left.\frac{2 \overline{\mathrm{V}}_{\mathrm{q}}^{2} \omega_{\mathrm{q}}}{\widetilde{\omega}_{q}}\right|_{T=T_{C}},
$$

$$
T_{C}=\Omega\left[k_{\beta} \tanh ^{-1}\left(\frac{4 \Omega}{\hat{J}}\right)\right]^{-1}
$$

\section{Results and Discussion}

By using following model values of physical quantities given by Lyddane et al. ${ }^{19}$, Samara ${ }^{29}$, Chaudhuri et al. ${ }^{30}$ and Zikai et al. ${ }^{31}$ : 
$\Omega=82 \mathrm{Cm}^{-1}, J=344 \mathrm{Cm}^{-1}, \quad J^{\prime}=440 \mathrm{Cm}^{-1}, \bar{V}_{i k}=25.56 \mathrm{Cm}^{-1}, \omega_{k}=153 \mathrm{Cm}^{-1}$, and $\mathrm{T}_{\mathrm{c}}=$ $123 \mathrm{~K}$. The temperature dependence of $\left\langle S_{q}^{Z}\right\rangle$ and $\left\langle S_{q}^{X}\right\rangle$ have been calculated for $\mathrm{KH}_{2} \mathrm{PO}_{4}(\mathrm{KDP})$ crystal are reported in Table1 and shown in Figure 1.

Table 1. Calculated values of $\left\langle S_{q}^{Z}>\right.$ and $\left\langle S_{q}^{X}>\right.$ for $\mathrm{KH}_{2} \mathrm{PO}_{4}(\mathrm{KDP})$ crystal for different values of $J^{\prime}$.

\begin{tabular}{ccccccc}
\hline \multicolumn{7}{c}{$\left\langle S_{q}^{L}\right\rangle$} \\
$\begin{array}{c}\text { Temperature } \\
(\mathrm{K})\end{array}$ & $J^{\prime}=J / 3$ & $J^{\prime}=4 J / 3$ & $\begin{array}{c}J^{\prime}=7 J / 3 \\
\text { Present study }\end{array}$ & $J^{\prime}=J / 3$ & $J^{\prime}=4 J / 3$ & $J^{\prime}=7 J / 3$ \\
\hline 20 & 0.500 & 0.499 & 0.499 & 0.454 & 0.369 & 0.311 \\
40 & 0.498 & 0.499 & 0.499 & 0.455 & 0.369 & 0.311 \\
60 & 0.459 & 0.481 & 0.498 & 0.460 & 0.376 & 0.312 \\
80 & 0.257 & 0.278 & 0.487 & 0.482 & 0.446 & 0.317 \\
100 & 0.137 & 0.143 & 0.454 & 0.489 & 0.479 & 0.332 \\
120 & 0.102 & 0.104 & 0.409 & 0.490 & 0.485 & 0.354 \\
122 & 0.100 & 0.100 & 0.403 & 0.491 & 0.486 & 0.357 \\
123 & 0.000 & 0.000 & 0.400 & 0.492 & 0.492 & 0.358 \\
125 & 0.000 & 0.000 & 0.398 & 0.484 & 0.484 & 0.449 \\
130 & 0.000 & 0.000 & 0.389 & 0.468 & 0.468 & 0.457 \\
135 & 0.000 & 0.000 & 0.373 & 0.446 & 0.446 & 0.443 \\
140 & 0.000 & 0.000 & 0.352 & 0.426 & 0.426 & 0.436 \\
145 & 0.000 & 0.000 & 0.320 & 0.408 & 0.408 & 0.410 \\
150 & 0.000 & 0.000 & 0.300 & 0.396 & 0.396 & 0.392 \\
\hline
\end{tabular}

In Figure 1, the curve ' $a$ ' is the case of $J$ ' $=J / 3<4 J / 3$, curve ' $b$ ' is $J^{\prime}=4 \mathrm{~J} / 3<7 \mathrm{~J} / 3$, curve 'c' is $J^{\prime}=7 \mathrm{~J} / 3>4 \mathrm{~J} / 3$. In curve 'a' and 'b' the value of $\left\langle S_{q}^{Z}>\right.$ increases to the saturated value 0.5 from zero, when temperature decreases from transition temperature. That is the case of second order phase transition. But in curve ' $c$ ' the change of $\left\langle s_{q}^{Z}>\right.$ with the temperature starts from a non-zero value $\left\langle s_{q}^{Z}>\right.$ at point A that is to say, when temperature decreases $\left\langle S_{q}^{Z}\right\rangle$ increases to the saturation value from the finite value of $\left\langle S_{q}^{Z}\right\rangle$. This is the case of first order phase transition. The temperature at point ' $\mathrm{A}$ ' is transition temperature (Tc), and the value of $\left\langle S_{q}^{Z}\right\rangle$ at 'A' is the discontinuity of $\left\langle S_{q}^{Z}\right\rangle$. The value of $\left\langle S_{q}^{X}>\right.$ decreases when temperature decreases in the ferroelectric phase. On the other hand, in paraelectric phase the value of $\left\langle S_{q}^{X}\right\rangle$ decreases when temperature increases from transition temperature. 


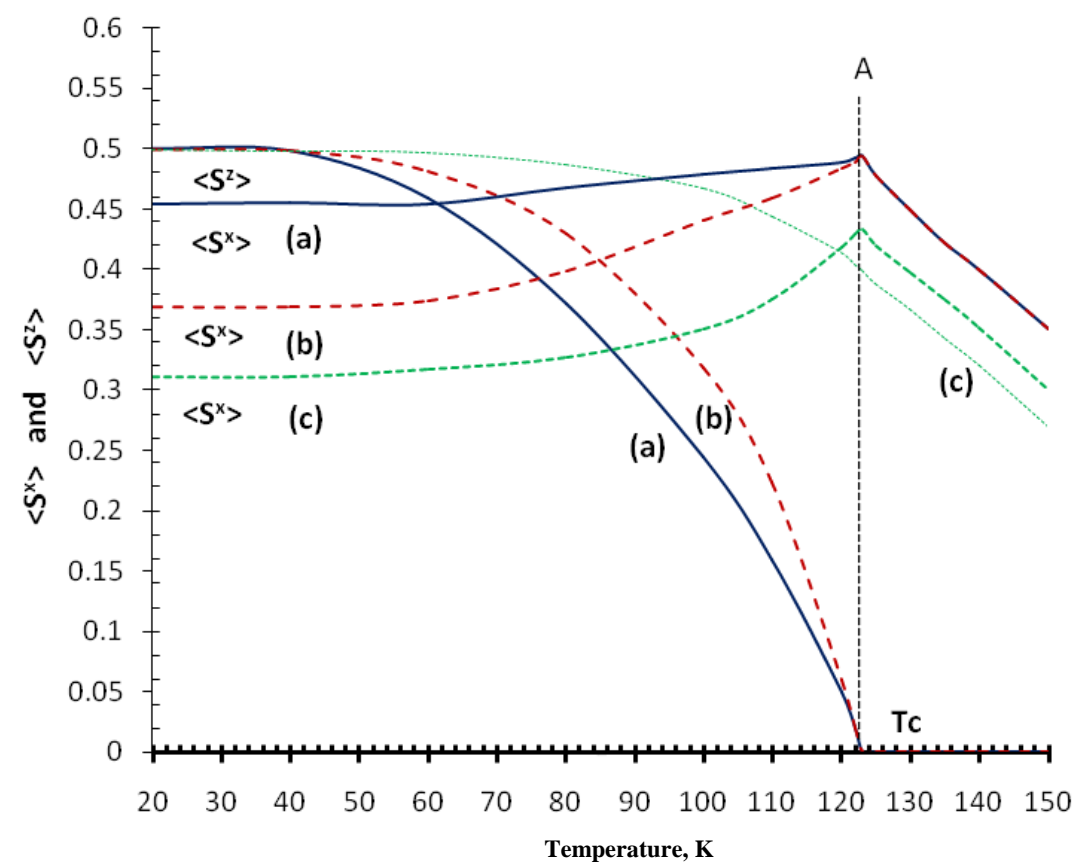

Figure 1. Temperature dependence of $\left\langle S_{q}^{Z}>\right.$ and $\left\langle S_{q}^{X}>\right.$ for $\mathrm{KH}_{2} \mathrm{PO}_{4}(\mathrm{KDP})$ crystal for different values of $J^{\prime}$. (i) $J^{\prime}=J / 3$, (ii) $J^{\prime}=4 J / 3$, and (iii) $J^{\prime}=7 J / 3$ (present study).

The Green's function method and Dyson's equation treatment, using model Hamiltonian, Equation 1; for hydrogen bonded ferroelectrics give the expression for collective mode frequency shifts and widths by considering all the possible interactions. In the paraelectric phase the temperature dependence of normalized collective phonon frequency enables one to calculate the transition temperature (Tc) Equation 10; and effective exchange coupling constant $\hat{J}$, Equation 9 ; which increases due to proton-phonon coupling and decreases due to anharmonic interactions. Thus the Green's function method with Dyson's equation treatment conveniently describes the transition properties of $\mathrm{KH}_{2} \mathrm{PO}_{4}(\mathrm{KDP})$ - system. The theoretical results are found in good agreement with the experimental results.

\section{Acknowledgement}

The authors wish to thank Dr K S Bartwal RRCAT, Indore, Dr O P Thakur, DRDO, New Delhi for the valuable suggestions and encouragement.

\section{References}

1. Busch G, Helv Phys Acta, 1938, 11, 269-298; DOI:10.5169/seals-110854

2. Mason W P, Phys Rev., 1946, 69, 173.

3. Jona F and Shirane G, Ferroelectric Crystals: Pergamon Press, 1962.

4. Kaminow I P and Damen T C, Phys Rev Lett., 1968, 20, 1105.

5. Tokunaga M and Matsubara T, Prog Theor Phys., 1966, 35, 581-599; DOI:10.1143/PTP.35.581

6. Kobayashi K K, J Phys Soc Japan, 1968, 24(3), 497-508; DOI:10.1143/JPSJ.24.497

7. Tokunaga M, Prog Theor Phys., 1984, 80, 156-162. 
8. Tominaga Y, Tokunaga $M$ and Tatsuzaki I, Ferroelectrics, 1985, 63(2), 171-178; DOI:10.1080/00150198508221398

9. Upadhyay T C and Semwal B S, Ind J Pure Appl Phys., 2002, 40, 615.

10. Upadhyay T C and Semwal B S, Pramana J Phys., 2003, 60(3), 525-533; DOI:10.1007/BF02706161

11. Upadhyay T C, Bhandari R S and Semwal B S, Pramana J Phys., 2006, 67(3), 547552; DOI:10.1007/s12043-006-0016-y

12. Deorani S C, Naithani U C and Semwal B S, Ind J Pure Appl Phys., 1999, 37, 215.

13. Deorani S C, Singh P and Yadav M S, Mat Sci Res India, 2011, 8(1), 137-147.

14. Blinc R and Zeks B, Ferroelectrics, 1987, 72(1), 193-227; DOI:10.1080/00150198708017947

15. Tokunaga $\mathrm{M}$ and Matsubara T, Ferroelectrics, 1987, 72(1), 175-191; DOI:10.1080/00150198708017946

16. Tokunaga $\mathrm{M}$ and Tatsuzaki I, Phase Trans., 1984, 4(2), 97-155; DOI:10.1080/01411598408220327

17. Blinc R, J PhysChem Solids, 1960, 13(3-4), 204-211; DOI:10.1016/00223697(60)90003-2

18. Kaminow I P and Damen T C, Phys Rev Lett., 1968, 20, 1105-1108.

19. Lyddane R H, Sachs R G and Teller E, Phys Rev., 1941, 59, 673.

20. Scott J F, Rev Mod Phys., 1974, 46, 83.

21. Tokunaga M and Matsubara T, Prog Theor Phys., 1966, 35(4), 581-599; DOI:10.1143/PTP.35.581

22. Gennes P G, Solid State Commun., 1963, 1(6), 132-137; DOI:10.1016/00381098(63)90212-6

23. Katiyar R S, Ryan J F and Scott J F, Phys Rev., 1971, B4, 2635; 1971, B4, 2685.

24. Lavrencic B B, Levstek I, Zeks B, Blinc R and Hadzi D, Chem Phys Lett., 1970, 5(7), 441-444; DOI:10.1016/0009-2614(70)80057-4

25. Pak K N, Phys Stat Sol., 1973, B60(1), 233-239; DOI:10.1002/pssb.2220600125

26. Panwar N S and Semwal B S, Ind J Pure Appl Phys., 1990, 28, 706.

27. Ramakrishana V and Tanaka T, Phys Rev., 1977, B16, 422.

28. Ganguli S Nath D and Chaudhuri B K, Phys Rev., 1980, B21, 2937.

29. Samara G A, Ferroelectrics, 1973, 5(1), 25-37; DOI:10.1080/00150197308235776

30. Chaudhuri B K, Ganguli Sand Nath D, Phys Rev., 1981, B23, 2308.

31. Zikai Q, Jinbo Z and Chunlei W, Ferroelectrics, 1988, 101, 164.

32. Maradudin A A ans Fein A E, Phys Rev., 1962, 128, 2589.

33. Blinc R and Zeks B, Adv Phys., 1972, 21(93), 693-757;

DOI:10.1080/00018737200101348 